UPRF-95-434

\title{
Adiabatic Motion of a Quantum Particle in a Two-Dimensional Magnetic Field
}

\author{
P. Maraner \\ Dipartimento di Fisica, Università di Parma, \\ and INFN, Gruppo collegato di Parma, \\ Viale delle Scienze, 43100 Parma, Italy
}

\begin{abstract}
The adiabatic motion of a charged, spinning, quantum particle in a two - dimensional magnetic field is studied. A suitable set of operators generalizing the cinematical momenta and the guiding center operators of a particle moving in a homogeneous magnetic field is constructed. This allows us to separate the two degrees of freedom of the system into a fast and a slow one, in the classical limit, the rapid rotation of the particle around the guiding center and the slow guiding center drift. In terms of these operators the Hamiltonian of the system rewrites as a power series in the magnetic length $l_{B}=\sqrt{\frac{\hbar c}{e B}}$ and the fast and slow dynamics separates. The effective guiding center Hamiltonian is obtained to the second order in the adiabatic parameter $l_{B}$ and reproduces correctly the classical limit.
\end{abstract}

short title: Adiabatic motion in a 2-dimensional magnetic field PACS: 03.65.-w, 02.90.+p 


\section{Introduction}

The study of the adiabatic motion of charged particles in slowly-varying magnetic fields is a very well developed subject of classical mechanics [1]. It is of utmost importance in plasma physics, in the study of particle motion in the geomagnetic field as well as in astrophysics. For these reasons several efforts have been devoted over the years in order to gain a deeper understanding of the formal structure underling the problem. In this manner the original method of directly subjecting the equations of motion to a standard perturbative treatment [1], has been supported by the works of C. S. Gardner [2], H. E. Mynich [3] and R. G. Littlejohn [4, 5, 6] developing Hamiltonian theory and yielding a systematic procedure allowing the construction of adiabatic invariants. While Gardner and Mynich based their approach on field lines coordinates and canonical transformations, Littlejohn proceeded by investigating the Hamiltonian structure of the system and by phase space (symplectic) geometrical techniques. In this paper we deal with the problem of the adiabatic motion of charged, spinning, quantum particle in a slowly-varying magnetic field. To this task we develop an algebraic technique which very much reminds the one used by Littlejohn in classical mechanics. Nevertheless we make use of a different set of variables and do not make explicit use of symplectic geometry. We restrict our attention to particles moving in a plane under influence of a time-independent perpendicular magnetic field. This is not only an expedient in order to illustrate our technique in a simple case. Although the planarity constraint may seems to be artificial in the classical context it is not in the quantum-mechanical one. Devices producing the confinement of electrons on a plane are in fact widely studied in physics, as example in the quantum Hall effect [7]. When these systems interacts with arbitrary inhomogeneous magnetic fields a realistic analysis indicates the effective dynamics to be described by a Hamiltonian proportional to the two-dimensional Laplace operator in minimal coupling with an effective magnetic field represented by the normal component of the original magnetic field [8]. This is the kind of system with which we are going to deal with. Our perturbative analysis is shown to be consistent with some general results concerning the ground state of quantum particles moving in an arbitrary two-dimensional magnetic fields [9, 10].

Before facing the quantum problem it is better to briefly recall the main features of the classical one. We start therefore by considering a classical particle of mass $m$ and charge $e$ moving in a plane under the influence of a normal uniform magnetic field of magnitude $B$. Let $\vec{x}=\left(x^{1}, x^{2}\right)$ denote the position of the particle and $\vec{v}=\left(v^{1}, v^{2}\right)$ its velocity. As well known the particle follows a circular orbit of radius $r_{B}=\frac{m c}{e B}|\vec{v}|$ which center remains motionless. The frequency of the motion is the cyclotron frequency $\omega_{B}=\frac{e B}{m c}$. The center of the orbit is called guiding center and its coordinates $\vec{X}=\left(X^{1}, X^{2}\right)$ are related to 
Figure 1: Adiabatic motion of a charged particle in a two-dimensional magnetic field.

the position and the velocity of the particle by the relation $X^{i}=x^{i}+\frac{m c}{e B} \varepsilon^{i k} v_{k}$. If The stronger the magnetic field the smaller is the radius of the orbit and faster the particle. By increasing the magnitude $B$ of the magnetic field, hence, the classical particle explores a portion of the plane which becomes smaller and smaller. This gives a reason of the why in classical mechanics the motion in a very strong magnetic field may be studied along the same lines of that in weakly inhomogeneous one. This fact is not immediate in quantum mechanics since even in a uniform magnetic field the wavefunctions are not localized by increasing the magnetic field.

Let us consider now the case of a weakly inhomogeneous magnetic field of magnitude $B(\vec{x})$. What happens is that the particle keeps on rapidly rotating around the guiding center $\vec{X}$ but, this time, the guiding center no longer remains motionless. It begins to slowly drift in the plane. The cyclotron frequency becomes, in addition, a function of the position $\omega_{B}(\vec{x}) \simeq \frac{e B(\vec{X})}{m c}$. We can therefore distinguish two way of move of the system: the very fast rotation around the guiding center and the slow drift of the guiding center in the plane. From the point of view of the Hamiltonian theory it is not immediate to understand how to deal with this situation. The systematic procedure for finding a proper set of canonical variables for the system has been given by Gardner [2] whereas Litteljohn [1] has shown how to construct these variables as power series in an appropriate adiabatic parameter 2 . The rotational motion of the particle may then be separated from that of the guiding center and the guiding center motion result to be described as the phase space

\footnotetext{
${ }^{1} \varepsilon_{i j}=\varepsilon^{i j}$ denotes the completely antisymmetric tensor in two dimensions: $\varepsilon_{11}=\varepsilon_{22}=0, \varepsilon_{12}=$ $-\varepsilon_{21}=1$. Everywhere in this paper the sum over repeated indexes is understood.

${ }^{2}$ In his work Lilletjohn made use of a set on noncanonical variables. This may however be reconduced to a canonical one by means of an appropriate transformation.
} 
motion of the remaining couple of canonical variables.

We now come to quantum mechanics. The first thing which hits us in the eye is that Plank's constants $\hbar$ introduces a length-scale into the problem: the magnetic length

$$
l_{B}=\sqrt{\frac{\hbar c}{e B}} .
$$

Quantum mechanics provides therefore an universal means to evaluate the order of magnitude of the variation of a magnetic field: the comparison between the length-scale over which the field varies and the magnetic length. For a rather weak 1 gauss magnetic field, $e$ the electron charge, $l_{B}$ is already a very small length, $l_{B} \simeq 10^{-4} \mathrm{~cm}$. The magnetic length $l_{B}$ appears therefore as a natural adiabatic parameter of the theory, that, on the contrary to the classical case, have not to be introduced by hand.

In this paper we construct a suitable set of operators allowing to rewrite the Hamiltonian describing the motion of a particle in a two-dimensional magnetic field as a formal power series in the magnetic length $l_{B}$. The expansion parameter having the dimension of a length, the perturbative series makes sense only when the $n$-th derivative of the field times $l_{B}{ }^{n}$ is a very small number in every point of the plane.

In section 2 we give an outlook to the anomalous Hamiltonian structure of a particle interacting with a uniform magnetic field. The construction of a set of operators adapting to this simple problem indicates clearly the presence of a fast and a slow degree of freedom of the system. It also suggests the way to be followed in the general case. In section 3 we construct a new set of variables $\left\{\Pi_{i}, X^{i} ; i=1,2\right\}$ adapting to the inhomogeneous problem. In the semiclassical picture the $\Pi_{i} \mathrm{~s}$ describe the rapid rotation of the particle, whereas the $X^{i}$ s take care of the dynamics of the guiding center. The construction is carried on order by order in the perturbative parameter $l_{B}$ up to the second order. In section 4 the cinematical momenta $\pi_{i}$ and the coordinates $x^{i}, i=1,2$, of the particle are rewritten in terms of the new variables. This allows to write down the Hamiltonian of the system as a power series in the magnetic length $l_{B}$. An appropriate unitary transformation is finally used in section 5 in order to make the perturbative expansion to depend on the variable $\Pi_{1}$ and $\Pi_{2}$ only by means of their combination $J=\frac{1}{2}\left(\Pi_{1}^{2}+\Pi_{2}^{2}\right)$. The fast rotational motion of the particle may so be separated from the slow drift of the guiding center. In the classical limit our result reproduces correctly Littlejohn's Hamiltonian [4]. Section 6 contains our conclusions. 


\section{An outlook to the canonical structure}

Magnetic interactions appear as modifications of the canonical structure of dynamical systems. This feature plays a central role in the Hamiltonian, and hence quantum, description of the adiabatic motion of charged particles in strong magnetic fields. We begin, therefore, by briefly reviewing this aspect of the problem in the case of a homogeneous magnetic field, introducing at the same time the conventions and the notations we use in the rest of the paper.

Let us consider a spin-1/2 particle of mass $m$, charge $e$ and gyromagnetic factor $g$ constrained to move in the $x^{1}-x^{2}$ plane in presence of the homogeneous magnetic field $\mathbf{B}(\vec{x})=B \hat{z}$. Denoting by $a_{i}(\vec{x}), i=1,2$, an arbitrary choice of vector potential for the dimensionless field $\mathbf{B}(\vec{x}) / B, \partial_{i} a_{j}-\partial_{j} a_{i}=\varepsilon_{i j}$, the dynamics of the particle is described by the Pauli Hamiltonian

$$
\mathcal{H}=\frac{1}{2 m} \sum_{i=1,2}\left(-i \hbar \frac{\partial}{\partial x^{i}}-\frac{e B}{c} a_{i}(\vec{x})\right)^{2}-g \frac{\hbar e B}{m c} \sigma_{3} .
$$

It is customary to parametrize the system by means of the canonical variables $p_{i}=$ $-i \hbar \partial / \partial x^{i}$ and $x^{i}, i=1,2$. Nevertheless, Hamiltonian (Z) takes an extremely simpler form if we replace the canonical momenta $p_{i}$ by the gauge covariant cinematical momenta $\pi_{i}, \pi_{i}=p_{i}-\frac{e B}{c} a_{i}(\vec{x})$. Aside from a scale factor $\pi_{1}$ and $\pi_{2}$ behaves as conjugate coordinates and Hamiltonian (2) rewrites as that of an harmonic oscillator, allowing the immediate solution of the problem. In order to get an adequate set of canonical variables for the description of the system, it is therefore more convenient to keep the cinematical momenta $\pi_{i}$ and to replace the coordinates $x^{i}$ by the guiding center operators $X^{i}, X^{i}=x^{i}+\frac{c}{e B} \varepsilon^{i j} \pi_{j}$. In the classical limit the $X^{i}$ s represent the coordinates of the center of the classical orbit. The set of operators $\left\{\pi_{i}, X^{i} ; i=1,2\right\}$ fulfills the commutation relations

$$
\begin{aligned}
& {\left[\pi_{i}, \pi_{j}\right]=i \frac{\hbar e B}{c} \varepsilon_{i j},} \\
& {\left[\pi_{i}, X^{j}\right]=0} \\
& {\left[X^{i}, X^{j}\right]=-i \frac{\hbar c}{e B} \varepsilon^{i j},}
\end{aligned}
$$

so that, up to some scale factors, $\pi_{i}$ and $X^{i}$ may be recognized as canonical variables. We note that the cinematical momentum $\pi_{1}$ and the guiding center coordinates $X^{2}$ behave now as "canonical coordinates" whereas the cinematical momentum $\pi_{2}$ and the guiding center coordinate $X^{1}$ correspond to the respective "canonical momenta". The presence 
of a homogeneous magnetic field produces a kind of rotation of the canonical structure, mixing up canonical momenta and coordinates in new canonical variables.

An outlook to the canonical commutation relations (3) allows to single out a second, very important, peculiarity of the variables $\pi_{i}$ and $X^{i}$. Thinking to Heisenberg's equations of motion we immediately realize that the temporal variation of the $\pi_{i} \mathrm{~s}$ is proportional to $B, \dot{\pi}_{i}=i\left[\mathcal{H}, \pi_{i}\right] / \hbar \simeq B$, whereas that of the $X^{i}$ s goes as $1 / B, \dot{X}^{i}=i\left[\mathcal{H}, X^{i}\right] / \hbar \simeq 1 / B$. p In the limit of very strong magnetic fields, the canonical structure of the system is distorted from the magnetic-scale $B$ and the $\pi_{i}$ s and $X^{i}$ s behave naturally as describing respectively a fast and a slow degree of freedom of the system.

In the next section we construct a set of operators generalizing the $\pi_{i}$ s and the $X^{i} \mathrm{~S}$ for an arbitrary inhomogeneous magnetic field $\mathbf{B}(\vec{x})=B b(\vec{x}) \hat{\mathbf{z}}$ (the dimension of the field being reabsorbed in the magnetic-scale $B$ ). This allows to set up an adiabatic description of the motion of a quantum particle in a two-dimensional magnetic field in a quite simple way.

For the shake of clearness it results to be convenient to introduce dimensionless quantities by factorizing the energy scale $\hbar \omega_{B}, \omega_{B}=\frac{e B}{m c}$, from the Hamiltonian. Recalling that the magnetic length $l_{B}=\sqrt{\frac{\hbar c}{e B}}$, we redefine the cinematical momenta as

$$
\pi_{i}=-i l_{B} \frac{\partial}{\partial x^{i}}-\frac{1}{l_{B}} a_{i}(\vec{x}) .
$$

Hamiltonian (2) writes then simply as $\mathcal{H}=\hbar \omega_{B}\left(\frac{1}{2} \sum \pi_{i}^{2}-g \sigma_{3}\right)$. It is instead necessary to keep guiding center operators with the dimension of a length,

$$
X^{i}=x^{i}+l_{B} \varepsilon^{i j} \pi_{j}
$$

in order to preserve the scale dependence of the canonical commutation relations (3)

$$
\begin{aligned}
& {\left[\pi_{i}, \pi_{j}\right]=i \varepsilon_{i j},} \\
& {\left[\pi_{i}, X^{j}\right]=0,} \\
& {\left[X^{i}, X^{j}\right]=-i l_{B}{ }^{2} \varepsilon^{i j} .}
\end{aligned}
$$

A general inhomogeneous magnetic field $\mathbf{B}(\vec{x})$ depends on coordinates with the dimension of a length, after all.

\footnotetext{
${ }^{3}$ Hamiltonian (2), of course, does not depend on the $X^{i}$ s. We nevertheless act as it does in order to explore the behaviour of the canonical variables in the limit of strong magnetic field.
} 


\section{Looking for a suitable set of variables}

We now consider a spin- $1 / 2$ particle of mass $m$, charge $e$ and gyromagnetic factor $g$ constrained to move on the $x^{1}-x^{2}$ plane under the action of the inhomogeneous magnetic field $\mathbf{B}(\vec{x})=B b(\vec{x}) \hat{\mathbf{z}} . b(\vec{x})$ is supposed to be an arbitrary positive nevervanishing smooth function of $\vec{x}$. Introducing an arbitrary choice of vector potential $a_{i}(\vec{x})$ for the dimensionless field $b(\vec{x}) \hat{\mathrm{z}}, \partial_{i} a_{j}-\partial_{j} a_{i}=\varepsilon_{i j} b(\vec{x})$, the system is again described by the Pauli Hamiltonian

$$
\mathcal{H}=\hbar \omega_{B}\left(\frac{1}{2} \sum_{i=1,2} \pi_{i}^{2}-g \sigma_{3} b(\vec{x})\right)
$$

where the cinematical momenta $\pi_{i}=-i l_{B} \partial_{i}-a_{i}(\vec{x}) / l_{B}$ have already been introduced in place of the canonical momenta $p_{i}$. In spite of the very simple dependence of Hamiltonian (17) on the $\pi_{i} \mathrm{~s}$, the cinematical momenta are no longer conjugate variables and can not be directly used to give a simple solution to the problem. The set of operators $\left\{\pi_{i}, x^{i} ; i=1,2\right\}$ fulfills in fact the commutation relations

$$
\begin{aligned}
& {\left[\pi_{i}, \pi_{j}\right]=i \varepsilon_{i j} b(\vec{x}),} \\
& {\left[\pi_{i}, x^{j}\right]=-i l_{B} \delta_{i}^{j},} \\
& {\left[x^{i}, x^{j}\right]=0 .}
\end{aligned}
$$

Nevertheless, we can try to construct a set of variables generalizing the cinematical momenta (4) and the guiding center operators (5) to the case of an inhomogeneous magnetic field as power series in the magnetic length $l_{B}$ with coefficients depending on the $\pi_{i} \mathrm{~s}$ and the $x^{i} \mathrm{~s}$. We proceed order by order in the parameter $l_{B}$ by constructing, say at the $n$-th order, a set of operators $\left\{\Pi_{i}^{(n)}, X_{(n)}^{i} ; i=1,2\right\}$ fulfilling adequate conditions. We require that

— in the limit of a constant magnetic field, $b(x) \rightarrow 1$, the $\Pi_{i}^{(n)}$ s and the $X_{(n)}^{i}$ s should reduce to the cinematical momenta (4) and to the guiding center operators (5) respectively,

— the $\Pi_{i}^{(n)}$ s should be conjugate variables up to terms of order $l_{B}{ }^{n}$,

— the $X_{(n)}^{i}$ s should commutate with the $\Pi_{i}^{(n)}$ s up to terms of order $l_{B}{ }^{n}$.

These commutation relations generalize obviously the (6). We nevertheless do not insist the $X_{(n)}^{i}$ s to be conjugate variables 4 . 


\section{Zero order variables}

In order to fulfil the conditions above up to terms of order $l_{B}$ we simply rescale the cinematical momenta $\pi_{i}$ by a factor $b^{-1 / 2}$,

$$
\Pi_{i}^{(0)}=\frac{1}{2}\left\{b^{-1 / 2}, \pi_{i}\right\}
$$

where the function $b^{-1 / 2}$ should be evaluated in $\vec{X}_{(0)} \equiv \vec{x}$. The anticommutator $\{$,$\} is$ introduced in order to make the $\Pi_{i}^{(0)}$ s hermitian. A brief computation gives the commutation relation fulfilled by the set of operators $\left\{\Pi_{i}^{(0)}, X_{(0)}^{i} ; i=1,2\right\}$,

$$
\begin{aligned}
& {\left[\Pi_{i}^{(0)}, \Pi_{j}^{(0)}\right]=i \varepsilon_{i j}+i \varepsilon_{i j} \frac{l_{B}}{4} \varepsilon^{k l}\left\{\frac{\left(\partial_{k} b\right)}{b^{3 / 2}}, \Pi_{l}^{(0)}\right\},} \\
& {\left[\Pi_{i}^{(0)}, X_{(0)}^{j}\right]=-i l_{B} \delta_{i}^{j} b^{-1 / 2}} \\
& {\left[X_{(0)}^{i}, X_{(0)}^{j}\right]=0}
\end{aligned}
$$

where all the functions have to be evaluated in $\vec{X}_{(0)}$. As required the $\Pi_{i}^{(0)}$ s result to be conjugate variables up to terms of order $l_{B}$ and the commutators between the $\Pi_{i}^{(0)} \mathrm{s}$ and the $X_{(0)}^{i}$ s are again of order $l_{B}$.

\section{First order variables}

We proceed by constructing a couple of operators $X_{(1)}^{i}, i=1,2$, commuting with the $\Pi_{i}^{(0)} \mathrm{s}$ up to terms of order $l_{B}^{2}$. The goal is achieved by performing a transformation generalizing (5) to the case of an inhomogeneous magnetic field. $X_{(1)}^{i}$ is defined as

$$
X_{(1)}^{i}=x^{i}+\frac{l_{B}}{2} \varepsilon^{i k}\left\{b^{-1 / 2}, \Pi_{k}^{(0)}\right\}
$$

the function $b^{-1 / 2}$ being evaluated in $\vec{X}_{(0)}$. We have so the following set of commutation relations

$$
\begin{aligned}
{\left[\Pi_{i}^{(0)}, \Pi_{j}^{(0)}\right]=} & i \varepsilon_{i j}+i \varepsilon_{i j} \frac{l_{B}}{4} \varepsilon^{k m}\left\{\frac{\left(\partial_{k} b\right)}{b^{3 / 2}}, \Pi_{m}^{(0)}\right\}+ \\
& -i \varepsilon_{i j} \frac{l_{B}{ }^{2}}{8} \varepsilon^{k m} \varepsilon^{l n}\left\{\frac{\left(\partial_{k} \partial_{l} b\right)}{b^{2}}-\frac{3}{2} \frac{\left(\partial_{k} b\right)\left(\partial_{l} b\right)}{b^{3}},\left\{\Pi_{m}^{(0)}, \Pi_{n}^{(0)}\right\}\right\}+o\left(l_{B}{ }^{3}\right),
\end{aligned}
$$




$$
\begin{aligned}
& {\left[\Pi_{i}^{(0)}, X_{(1)}^{j}\right]=i l_{B}{ }^{2} \varepsilon^{j k}\left(\frac{1}{2}\left\{\frac{\left(\partial_{i} b\right)}{b^{2}}, \Pi_{k}^{(0)}\right\}-\frac{1}{4}\left\{\frac{\left(\partial_{k} b\right)}{b^{2}}, \Pi_{i}^{(0)}\right\}\right),} \\
& {\left[X_{(1)}^{i}, X_{(1)}^{j}\right]=-i l_{B}{ }^{2} \varepsilon^{i j} b^{-1}+o\left(l_{B}{ }^{3}\right) .}
\end{aligned}
$$

All the functions have to be evaluated in $\vec{X}_{(1)}$. We observe that the $X_{(1)}^{i}$ s are no longer commuting variables so that the evaluation of the magnetic field function $b$ and of its derivatives in $\vec{X}_{(1)}$ involves ordering ambiguities. The commutator of the $X_{(1)}^{i}$ being of order $l_{B}{ }^{2}$, these ambiguities concerns the terms $o\left(l_{B}{ }^{3}\right)$.

The operators $\Pi_{i}^{(1)} \mathrm{s}$ are constructed by adding a correction of order $l_{B}$ to the $\Pi_{i}^{(0)} \mathrm{s}$. This correction is looked for as a homogeneous second order polynomial in the $\Pi_{i}^{(0)} \mathrm{s}$. A brief computation indicates that the right choice is

$$
\Pi_{i}^{(1)}=\Pi_{i}^{(0)}-\frac{l_{B}}{24} \varepsilon^{k m}\left\{\frac{\left(\partial_{k} b\right)}{b^{3 / 2}},\left\{\Pi_{i}^{(0)}, \Pi_{m}^{(0)}\right\}\right\}
$$

where the functions are evaluated in $\vec{X}_{(1)}$. The commutation relations fulfilled by the set of operators $\left\{\Pi_{i}^{(1)}, X_{(1)}^{i} ; i=1,2\right\}$ can be immediately obtained by means of (12)

$$
\begin{aligned}
& {\left[\Pi_{i}^{(1)}, \Pi_{j}^{(1)}\right]=i \varepsilon_{i j}-i \varepsilon_{i j} \frac{l_{B}{ }^{2}}{4} \varepsilon^{k m} \varepsilon^{l n}\left\{\frac{1}{2} \frac{\left(\partial_{k} \partial_{l} b\right)}{b^{2}}-\frac{5}{9} \frac{\left(\partial_{k} b\right)\left(\partial_{l} b\right)}{b^{3}},\left\{\Pi_{m}^{(1)}, \Pi_{n}^{(1)}\right\}\right\}+o\left(l_{B}{ }^{3}\right)} \\
& {\left[\Pi_{i}^{(1)}, X_{(1)}^{j}\right]=i l_{B}{ }^{2} \varepsilon^{j k}\left(\frac{1}{2}\left\{\frac{\left(\partial_{i} b\right)}{b^{2}}, \Pi_{k}^{(1)}\right\}-\frac{1}{4}\left\{\frac{\left(\partial_{k} b\right)}{b^{2}}, \Pi_{i}^{(1)}\right\}\right)} \\
& {\left[X_{(1)}^{i}, X_{(1)}^{j}\right]=-i l_{B}{ }^{2} \varepsilon^{i j} b^{-1}+o\left(l_{B}{ }^{3}\right) .}
\end{aligned}
$$

The $\Pi_{i}^{(1)}$ s behave as conjugate variables up to terms of order $l_{B}{ }^{2}$ and the commutators between the $\Pi_{i}^{(1)} \mathrm{s}$ and the $X_{(1)}^{i} \mathrm{~s}$ vanish again up to second order terms in $l_{B}$.

\section{Second order variables}

The second and further order variables have to be constructed along the same line of the previous one. Looking for the $l_{B}{ }^{2}$-correction to be added to the $X_{(1)}^{i}$ s as a homogeneous second order polynomial in the $\Pi_{i}^{(1)} \mathrm{s}$ we easily find

$$
X_{(2)}^{i}=X_{(1)}^{i}-\frac{l_{B}{ }^{2}}{8} \varepsilon^{i k} \varepsilon^{j l}\left\{\frac{\left(\partial_{j} b\right)}{b^{2}},\left\{\Pi_{k}^{(1)}, \Pi_{l}^{(1)}\right\}\right\},
$$


the functions being evaluated in $\vec{X}_{(1)}$. The relations (14) allow to compute the commutations relations

$$
\begin{aligned}
& {\left[\Pi_{i}^{(1)}, \Pi_{j}^{(1)}\right]=i \varepsilon_{i j}-i \varepsilon_{i j} \frac{l_{B}{ }^{2}}{4} \varepsilon^{k m} \varepsilon^{l n}\left\{\frac{1}{2} \frac{\left(\partial_{k} \partial_{l} b\right)}{b^{2}}-\frac{5}{9} \frac{\left(\partial_{k} b\right)\left(\partial_{l} b\right)}{b^{3}},\left\{\Pi_{m}^{(1)}, \Pi_{n}^{(1)}\right\}\right\}+o\left(l_{B}^{3}\right)} \\
& {\left[\Pi_{i}^{(1)}, X_{(2)}^{j}\right]=0+o\left(l_{B}^{3}\right)} \\
& {\left[X_{(2)}^{i}, X_{(2)}^{j}\right]=-i l_{B}{ }^{2} \varepsilon^{i j} b^{-1}+o\left(l_{B}{ }^{3}\right)}
\end{aligned}
$$

where every function is now evaluated in $\vec{X}_{(2)}$. Finally we proceed by constructing $\Pi_{i}^{(2)}$ by adding an adequate homogeneous third order polynomial in the $\Pi_{i}^{(1)} \mathrm{s}$ to $\Pi_{i}^{(1)}$,

$$
\Pi_{i}^{(2)}=\Pi_{i}^{(1)}+\frac{l_{B}{ }^{2}}{4} \varepsilon^{k m} \varepsilon^{l n}\left\{\frac{1}{2} \frac{\left(\partial_{k} \partial_{l} b\right)}{b^{2}}-\frac{5}{9} \frac{\left(\partial_{k} b\right)\left(\partial_{l} b\right)}{b^{3}}, \Pi_{m}^{(1)} \Pi_{i}^{(1)} \Pi_{n}^{(1)}\right\},
$$

the function $b$ and its derivatives being again evaluated in $\vec{X}_{(2)}$. The set of operators $\left\{\Pi_{i}^{(2)}, X_{(2)}^{i} ; i=1,2\right\}$ fulfills the desired commutation relations

$$
\begin{aligned}
& {\left[\Pi_{i}^{(2)}, \Pi_{j}^{(2)}\right]=i \varepsilon_{i j}+o\left(l_{B}{ }^{3}\right),} \\
& {\left[\Pi_{i}^{(2)}, X_{(2)}^{j}\right]=0+o\left(l_{B}{ }^{3}\right),} \\
& {\left[X_{(2)}^{i}, X_{(2)}^{j}\right]=-i l_{B}{ }^{2} \varepsilon^{i j} b^{-1}+o\left(l_{B}{ }^{3}\right) .}
\end{aligned}
$$

The $\Pi_{i}^{(2)} \mathrm{s}$ are conjugate up to terms of order $l_{B}{ }^{3}$ and commutate with the $X_{(2)}^{i} \mathrm{~s}$ again up to the third order in $l_{B}$. In the limit of a constant magnetic filed, of course, all the variables that we have introduced have the correct behaviour.

\section{A noncanonical set of variables}

In principle we may think to repeat this procedure an arbitrary number of times and to construct, as power series in $l_{B}$ with coefficients polynomial in the $\pi_{i}$ s and depending on the $x^{i}$ s through the function $b$ and its derivatives, a set noncanonical operators $\left\{\Pi_{i}, X^{i} ; i=\right.$ $1,2\}$ fulfilling the commutation relations

$$
\begin{aligned}
& {\left[\Pi_{i}, \Pi_{j}\right]=i \varepsilon_{i j},} \\
& {\left[\Pi_{i}, X^{j}\right]=0,} \\
& {\left[X^{i}, X^{j}\right]=-i l_{B}^{2} \varepsilon^{i j} b^{-1},}
\end{aligned}
$$


$b^{-1}$ being now evaluated in $\vec{X}$. Anyway, in order to discuss the problem to the second order in the adiabatic parameter $l_{B}$, we only need to know the first three terms of these series, terms which we have already evaluated. Up to terms of order $l_{B}{ }^{3}$ we may therefore confuse the $\Pi_{i}^{(2)}$ s and $X_{(2)}^{i}$ s with the $\Pi_{i}$ s and $X^{i}$ s respectively.

Let us observe now, that aside from the function $b^{-1}$ appearing in the right-hand side of the third identity, the commutation relations (19) correspond to the (6). Although the $X^{i}$ s are not conjugate variables they commute with the $\Pi_{i}$ s and their commutator is a function of the $X^{i}$ s alone. The Hilbert space of the system, separates therefore under the action of the two couple of operators in the direct sum of two subspaces describing each one a degree of freedom of the system. The scale dependence of the commutation relations (19) indicates again that in the limit of very strong magnetic fields ( small $l_{B}$ ) the $\Pi_{i}$ s and the $X^{i}$ s describe respectively a fast and a slow degree of freedom of the system. The adiabatic cinematical momenta $\Pi_{i}$ and the adiabatic guiding center operators $X^{i}$ introduced in this section, appear therefore as a suitable choice of variables for our problem.

\section{The adiabatic expansion}

The next steep is to rewrite Hamiltonian (17) in terms of the new set of variables. To this purpose we have first to invert the power series expressing the $\Pi_{i} \mathrm{~s}$ and the $X^{i} \mathrm{~s}$ in terms of the $\pi_{i}$ s and the $x^{i} \mathrm{~s}$ and then to replace these expressions in (7). As a result the Hamiltonian will appear as a power series in the magnetic length $l_{B}$

$$
\mathcal{H}=\mathcal{H}^{(0)}+l_{B} \mathcal{H}^{(1)}+l_{B}^{2} \mathcal{H}^{(2)}+\ldots
$$

In the limit of slowly-varying magnetic field, that is when the magnetic length may be considered small with respect to the length-scale over which the magnetic fields varies, this equation may be interpreted as a perturbative expansion of the hamiltonian and used to get approximate expressions of the spectrum and the wavefunctions of the system. We call it the adiabatic expansion of the Hamiltonian.

The task of obtaining the operators $\pi_{i}$ and $x^{i}$ in terms of the new variables $\Pi_{i}$ and $X^{i}$ is not a hard one. Recalling that $X^{i}=X_{(2)}^{i}+o\left(l_{B}{ }^{3}\right)$ and using equations (9), (11), (13) and (15) we can immediately obtain the first three terms of the power series expressing the $x^{i} \mathrm{~s}$ in terms of the $\pi_{i} \mathrm{~s}$ and the $X^{i} \mathrm{~s}, x^{i}=x^{i}(\vec{\pi}, \vec{X})$. This allows to rewrite the $\Pi_{i}^{(0)} \mathrm{s}$ as functions of the the $\pi_{i}$ s and the $X^{i}$ s, so that by using equations (13), (17) and the relation $\Pi_{i}=\Pi_{i}^{(2)}+o\left(l_{B}^{3}\right)$, is possible to rewrite the $\Pi_{i}$ s as power series in $l_{B}$ with coefficients depending on the $\pi_{i}$ s and the $X^{i} \mathrm{~s}, \Pi_{i}=\Pi_{i}(\vec{\pi}, \vec{X})$. Inverting these series order by order 
we can finally calculate the $\pi_{i} \mathrm{~s}$ as functions of the $\Pi_{i} \mathrm{~s}$ and the $X^{i} \mathrm{~s}$. By substituting these expressions in $x^{i}=x^{i}(\vec{\pi}, \vec{X})$, we also get the $x^{i}$ s as functions of the $\Pi_{i}$ s and the $X^{i}$ s. A few computations conduce to the result

$$
\begin{aligned}
\pi_{i}= & b^{1 / 2} \Pi_{i}-\frac{l_{B}}{6} \frac{\left(\partial_{k} b\right)}{b} \varepsilon^{k m}\left\{\Pi_{i}, \Pi_{m}\right\}+ \\
& +l_{B}^{2}\left(\frac{1}{8} \frac{\left(\partial_{k} \partial_{l} b\right)}{b^{3 / 2}}-\frac{7}{72} \frac{\left(\partial_{k} b\right)\left(\partial_{l} b\right)}{b^{5 / 2}}\right) \varepsilon^{k m} \varepsilon^{l n} \Pi_{m} \Pi_{i} \Pi_{n}+o\left(l_{B}^{3}\right), \\
x^{i}= & X^{i}-l_{B} \varepsilon^{i k} b^{-1 / 2} \Pi_{k}-\frac{l_{B}^{2}}{12} \varepsilon^{i k} \varepsilon^{j l} \frac{\left(\partial_{j} b\right)}{b^{2}}\left\{\Pi_{k}, \Pi_{l}\right\}+o\left(l_{B}{ }^{3}\right)
\end{aligned}
$$

the function $b$ and its derivatives being evaluated in $\vec{X}$. As a check we can easily reobtain the commutation relations (8) by means of the expressions (21), (22) and (19).

The first three terms of the adiabatic expansion of the Hamiltonian can now be immediately obtained by substituting the expressions (21) and (22) in (7). In order to simplify the notation we introduce the operators $J=\frac{1}{2} \delta^{i j} \Pi_{i} \Pi_{j}, J_{k}=\frac{1}{2} \delta^{i j} \Pi_{i} \Pi_{k} \Pi_{j}$ and $J_{k l}=\frac{1}{2} \delta^{i j} \Pi_{i}\left\{\Pi_{k}, \Pi_{l}\right\} \Pi_{j} . J$ is just the Hamiltonian of an harmonic oscillator in the conjugate variables $\Pi_{1}, \Pi_{2}$ and, as we will see in the next section, plays a central role in our discussion. A brief computation yields

$$
\begin{aligned}
\mathcal{H}^{(0)} / \hbar \omega_{B}= & b\left(J+g \sigma_{3}\right) \\
\mathcal{H}^{(1)} / \hbar \omega_{B}= & -\frac{\left(\partial_{i} b\right)}{b^{1 / 2}} \varepsilon^{i k}\left(2 J_{k} / 3+g \sigma_{3} \Pi_{k}\right) \\
\mathcal{H}^{(2)} / \hbar \omega_{B}= & \left(\frac{1}{8} \frac{\left(\partial_{i} \partial_{j} b\right)}{b}-\frac{3}{72} \frac{\left(\partial_{i} b\right)\left(\partial_{j} b\right)}{b^{2}}\right) \varepsilon^{i k} \varepsilon^{j l}\left(J_{k l}+2 g \sigma_{3}\left\{\Pi_{k}, \Pi_{l}\right\}\right)+ \\
& -\left(\frac{1}{16} \frac{\left(\partial_{i} \partial_{j} b\right)}{b}-\frac{5}{144} \frac{\left(\partial_{i} b\right)\left(\partial_{j} b\right)}{b^{2}}\right) \delta^{i j} \\
\ldots & .
\end{aligned}
$$

These equations represent a good starting point for a perturbative analysis of the system. Nevertheless there is still some more work that may be done in order to make this task simpler. In the next section we will follow a strategy which very much recalls the one used in the perturbation theory of Hamiltonian mechanics, in order to find a unitary transformation making $\mathcal{H}$ to depend on $\Pi_{1}$ and $\Pi_{2}$ only by means of their combination $J$ and its powers. $J$ results then into an adiabatic invariant which may be identified with magnetic moment of gyration of the particle. 


\section{$5 \quad$ Effective guiding center dynamics}

A well known strategy in treating perturbative problems in classical mechanics is that of subjecting the the system to a series of near-identity canonical transformations in order to make the various orders of the perturbative expansion of the Hamiltonian to be independent on certain variables. In this way is possible to individuate the constants of motion of the system up to an arbitrary order in the perturbative parameter. The approximate solution of the problem results then notably simplified. In this section we will adopt the equivalent technique in quantum mechanics. Hamiltonian (20) will be subjected to a near-identity unitary transformation such that the following criteria is fulfilled: the new Hamiltonian should depend on the conjugate variables $\Pi_{1}$ and $\Pi_{2}$ only by means of the operator $J$ and its powers. In this way $J$ results into a constant of motion of the system and the dynamics of the $X^{i} \mathrm{~s}$ separates from that of the $\Pi_{i} \mathrm{~s}$.

Let us therefore consider a near-identity unitary operator $U$, represented in the form $U=\mathrm{e}^{i \mathcal{L}}$. The hermitian operator $\mathcal{L}$ will differ from the identity operator $\mathbf{1}$ by terms of order grater than $l_{B}$ so that we may represent it as the power series

$$
\mathcal{L}=\mathbf{1}+l_{B} \mathcal{L}^{(1)}+l_{B}{ }^{2} \mathcal{L}^{(2)}+\ldots
$$

Subjecting Hamiltonian (20) to this transformation produces the result

$$
\begin{aligned}
& \mathrm{e}^{i \mathcal{L}} \mathcal{H} \mathrm{e}^{-i \mathcal{L}}=\mathcal{H}^{(0)}+l_{B}\left(\mathcal{H}^{(1)}+i\left[\mathcal{L}^{(1)}, \mathcal{H}^{(0)}\right]\right)+ \\
& \quad+l_{B}{ }^{2}\left(\mathcal{H}^{(2)}+i\left[\mathcal{L}^{(2)}, \mathcal{H}^{(0)}\right]+i\left[\mathcal{L}^{(1)}, \mathcal{H}^{(1)}+\frac{i}{2}\left[\mathcal{L}^{(1)}, \mathcal{H}^{(0)}\right]\right]\right)+\ldots
\end{aligned}
$$

The zero order term of the Hamiltonian remains unchanged whereas the others are corrected by additive terms depending on the commutators of the $\mathcal{L}^{(n)}$ s with $\mathcal{H}^{(0)}$ and the subsequent terms of the adiabatic expansion. We start therefore by looking for an operator $\mathcal{L}^{(1)}$ such that its commutator with $\mathcal{H}^{(0)},\left[\mathcal{L}^{(1)}, \mathcal{H}^{(0)}\right]$, might correct the first order term of the adiabatic expansion in the desired way. A brief computation indicates that it is possible to annihilate the first order term of the transformed Hamiltonian by choosing

$$
\mathcal{L}^{(1)}=-\frac{\left(\partial_{i} b\right)}{b^{1 / 2}} \delta^{i k}\left(2 J_{k} / 3+g \sigma_{3} \Pi_{k}\right) .
$$

The next steep is to construct the operator $\mathcal{L}^{(2)}$ making the second order term of the adiabatic expansion, $\mathcal{H}^{(2)}+i\left[\mathcal{L}^{(2)}, \mathcal{H}^{(0)}\right]+i\left[\mathcal{L}^{(1)}, \mathcal{H}^{(1)}\right] / 2$, to depend on $\Pi_{1}, \Pi_{2}$, only by means of their combination $J$ and its powers. The correct choice results to be

$$
\mathcal{L}^{(2)}=\frac{1}{16}\left(\frac{\left(\partial_{i} \partial_{j} b\right)}{b^{2}}-\frac{1}{3} \frac{\left(\partial_{i} b\right)\left(\partial_{j} b\right)}{b^{3}}\right) \varepsilon^{i k} \delta^{j l}\left(J_{k l}+2 g \sigma_{3}\left\{\Pi_{k}, \Pi_{l}\right\}\right)
$$


Having obtained further terms of the adiabatic expansion $(20)$ it would be possible to keep on this procedure to an arbitrary order. At least in principle therefore is possible to make $J$ into a constant of motion to an arbitrary order in the adiabatic parameter $l_{B}$. Our analysis stops to the second order. Subjecting Hamiltonian $(20)$, with $\mathcal{H}^{(0)}, \mathcal{H}^{(1)}$ and $\mathcal{H}^{(2)}$ given respectively by (23), (24) and (25), to the unitary transformation already described we get the new adiabatic expansion

$$
\begin{aligned}
\mathcal{H}^{\prime} / \hbar \omega_{B}=b\left(J+g \sigma_{3}\right)+\frac{l_{B}{ }^{2}}{4} & {\left[\frac{\triangle b}{b}-3 \frac{|\nabla b|^{2}}{b^{2}}\right]\left(J^{2}+2 g \sigma_{3} J\right)+} \\
+ & \frac{l_{B}^{2}}{16}\left[\frac{\triangle b}{b}-\left(1+8 g^{2}\right) \frac{|\nabla b|^{2}}{b^{2}}\right]+o\left(l_{B}{ }^{3}\right)
\end{aligned}
$$

where the functions $b, \triangle b=\delta^{i j}\left(\partial_{i} \partial_{j} b\right)$ and $|\nabla b|^{2}=\delta^{i j}\left(\partial_{i} b\right)\left(\partial_{j} b\right)$ are again evaluated in $\vec{X}$. Up to terms of order $l_{B}{ }^{3}$ the operator $J$ results into a constant of motion of the system and the harmonic oscillator degree of freedom described by $\Pi_{1}$ and $\Pi_{2}$ separates from the the guiding center motion described by $X^{1}$ and $X^{2}$. In the semiclassical picture the fast rotation of the particle around the guiding center corresponds to the phase space motion in the plane $\Pi_{1}-\Pi_{2}$ whereas the slow drift of the guiding center in the plane corresponds the phase space motion in the plane $X^{1}-X^{2}$. The adiabatic invariant $J$ may therefore be identified with the magnetic moment of gyration of the particle, that is the magnetic moment of the current loop described by the particle in one cyclotron gyration. In the classical limit the Hamiltonian operator (30) reproduces correctly Littlejohn's guiding center Hamiltonian [4].

Once the system has been frozen in one of its gyrating eigenstates, that is, in an eigenstate of the fast degree of freedom, Hamiltonian (30) describes the corresponding effective guiding center dynamics. It is interesting to observe that the zero order effective guiding center Hamiltonian corresponds to the magnetic field function $b(\vec{x})$ in which the coordinates $x^{1}$ and $x^{2}$ have been substituted with the noncommuting operators $X^{1}$ and $X^{2}$. Introducing a pair of Euler potentials $\mathrm{x}^{1}(\vec{x})$ and $\mathrm{x}^{2}(\vec{x})$ for the magnetic field $b(\vec{x}) \hat{\mathrm{z}}$, $\nabla \mathrm{x}^{1} \wedge \nabla \mathrm{x}^{2}=b$ [11], it is also possible to make the $X^{i}$ s into a couple of conjugate operators. Defining $\mathrm{X}^{1}=\mathrm{x}^{1}(\vec{X})$ and $\mathrm{X}^{2}=\mathrm{x}^{2}(\vec{X})$ we get in fact $\left[\mathrm{X}^{1}, \mathrm{X}^{2}\right]=-i l_{B}^{2}$, [2, 4. In order to evaluate the zero order spectrum of the system it is therefore sufficient to construct the Hamiltonian operator of the system by substituting the commuting variables $\mathrm{x}^{1}$ and $\mathrm{x}^{2}$ with the couple of conjugate operators $\mathrm{X}^{1}$ and $\mathrm{X}^{2}$ in the function $b\left(\vec{x}\left(\mathrm{x}^{1}, \mathrm{x}^{2}\right)\right)$. This is similar to a quantization procedure, which for some aspects, reminds the one explored by J. R. Klauder [12, 13]. 
Further corrections to the spectrum may be evaluated by means of standard perturbation theory and the subsequent terms of the adiabatic expansion (30).

To conclude the section we specialize to the case of electrons, $g=-1 / 2$, in order to check our result to be consistent with a theorem of Y. Aharonov and A. Casher stating, among other things, that the ground state of an electron moving in an arbitrary twodimensional magnetic field has always zero energy [9]. By setting the gyromagnetic factor to $-1 / 2$ our Hamiltonian reduces to the quite compact form

$$
\mathcal{H}^{\prime} / \hbar \omega_{B}=b\left(J-\frac{1}{2} \sigma_{3}\right)+\frac{l_{B}{ }^{2}}{4}\left[\frac{\triangle b}{b}-3 \frac{|\nabla b|^{2}}{b^{2}}\right]\left(J^{2}-\sigma_{3} J+\frac{1}{4}\right)+o\left(l_{B}{ }^{3}\right) .
$$

Recalling that the eigenvalues of $J$ are $E_{n}=\left(n+\frac{1}{2}\right), n=0,1,2 \ldots$, and that of $\sigma_{3}$ are $s= \pm 1$ it is immediate to observe that the ground sate energy of Hamiltonian (31), $(n=0, s=1)$, is zero up to terms of order $l_{B}{ }^{3}$.

\section{Discussion and conclusion}

The construction of a set of noncanonical operators sharing the main properties of the cinematical momenta and the guiding center operators of a particle moving in a homogeneous magnetic field, allows to separate the unperturbed dynamics of a quantum particle moving in a two-dimensional weakly-inhomogeneous magnetic field, from the perturbation making the guiding center dynamics nontrivial. The effective guiding center Hamiltonian writes furthermore as a power series in the magnetic length $l_{B}$. The main features of the construction are that it is perturbative in nature, that is, it is performed order by order in the adiabatic parameter $l_{B}$, and involves only simple algebraic manipulations. These properties allows to achieve the goal in a very simple and economical way.

It is worth-while to spend some words on the efficiency of the adiabatic expansion (30). As we said in the introduction the presence of a magnetic field in a quantum context introduces the length-scale $l_{B}$. Intuitively, we may therefore consider a magnetic field to be slowly-varying (weakly inhomogeneous), when its variation ratio over the magnetic lengthscale $l_{B}$ is a small number. The adiabatic expansion obtained in this paper completely confirms this picture. Furthermore, still for a rather week magnetic field of 1 gauss, the magnetic length is very small, $l_{B} \simeq 10^{-4} \mathrm{~cm}$. To have one's eye on some number, we recall that the geomagnetic field near to the surface of the earth is of the order of the gauss as well as the magnetic field produced by a wire in which flows a current of a few ampere. In the physics of the quantum Hall effect much stronger fields are used, typically of the 
order of $10^{4}-10^{5}$ gauss. All these fields varies significantly over lengths which go from the millimeter to some meters. That is to say, the adiabatic approximation is a very good one. Among the other things, therefore, our work gives a further explanation of the why so sharp Landau levels are observed in the quantum Hall effect.

Once the system has been frozen in one of its gyrating eigenstates, the dynamics of the remaining degree of freedom is described by means of a Hamiltonian operator which have to be constructed by quantizing the classical Hamiltonian $b\left(\vec{x}\left(\mathrm{x}^{1}, \mathrm{x}^{2}\right)\right)$ (replacing $\hbar$ with $l_{B}{ }^{2}$ ). The $l_{B}{ }^{2}$-terms of the adiabatic expansion (30) will in general contribute a small correction to the spectrum of the system. By an appropriate choice of the magnetic field, therefore, an arbitrary one-degree-of-freedom Hamiltonian may be reproduced. It would be interesting to explore the possibility of concretely realize devices of this kind.

We conclude by observing that the method we developed in this paper works in the full three-dimensional case as well as in the two-dimensional one. The study of the adiabatic motion of a charged, spinning, quantum particle in a three-dimensional magnetic field will be reported upon in a forthcoming publication.

\section{Aknoledgments}

I wish to warmly thank R. Ragazzon for very useful discussions.

\section{References}

[1] T. G. Northrop, The Adiabatic Motion of Charged Particles Interscience, N. Y. (1963)

[2] C. S. Gardner, Phys. Rev. 115, 791 (1959)

[3] H. E. Mynich , Phys. Rev. Lett. 43, 1019 (1979)

[4] R. G. Littlejohn, J. Math. Phys. 12, 2445 (1979)

[5] R. G. Littlejohn, Phys. Fluids. 24, 1730 (1981)

[6] R. G. Littlejohn, in Contemporary Mathematics Vol.28, 151 J. E. Marsden ed., American Mathematical Society (1984)

[7] R. E. Prange and S. M. Girvin ed., The Quantum Hall Effect Springer, Berlin (1989)

[8] M. Ikegami and Y. Nagaoka, Prog. Theo. Phys. Suppl.106, 235 (1991) 
[9] Y. Aharonov and A. Casher, Phys. Rev. A19, 2461 (1979)

[10] P. Maraner, Mod. Phys. Lett. A7 2555 (1992)

[11] D. P. Stern, Am. J. Phys. 38, 494 (1970)

[12] J. R. Kaluder, Ann. Phys. 188, 1201988

[13] J. R. Klauder and E. Onofri, Int. J. Mod. Phys. A4, 3939 (1989) 